\title{
Hypoxia induces hemorrhagic transformation in pituitary adenomas via the HIF-1 $\alpha$ signaling pathway
}

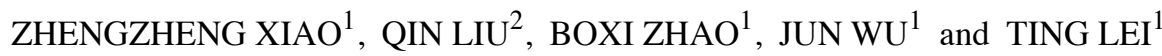 \\ ${ }^{1}$ Department of Neurosurgery, Tongji Hospital, ${ }^{2}$ Department of Pediatrics, Union Hospital, Tongji \\ Medical College, Huazhong University of Science and Technology, Wuhan 430030, P.R. China
}

Received May 31, 2011; Accepted July 11, 2011

DOI: 10.3892/or.2011.1416

\begin{abstract}
The hypoxia inducible factor $1 \alpha$ (HIF-1 $\alpha$ ) activity has been associated with various hemorrhagic events. The biological role of HIF-1 $\alpha$ in the hemorrhagic transformation of pituitary adenomas remains unknown. We hypothesized that fast growing tumor cells tend to predispose themselves to sublethal hypoxia and activate the HIF-1 $\alpha$ signaling pathway, leading to hemorrhagic transformation in pituitary adenomas. Here, we used apoplectic and non-apoplectic pituitary adenomas to determine the involvement of HIF-1 $\alpha$ signaling in intratumoral hemorrhage. We employed HIF-1 $\alpha$ overexpression/knockdown strategies to examine the association between HIF-1 $\alpha$ signaling and hemorrhagic presentation in vitro and in vivo. In support of our hypothesis, compared with nonhemorrhagic pituitary adenomas, higher cellular proliferation was observed in hemorrhagic ones and it correlated with increased HIF- $1 \alpha$ signaling. HIF-1 $\alpha$ overexpression activated its downstream genes, vascular endothelial growth factor and the proapoptotic BNIP3, in MMQ pituitary adenoma cells and this up-regulation was attenuated by HIF-1 siRNA. In vivo studies using MMQ cell xenografts in nude mice showed that HIF-1 $\alpha$ overexpression significantly promoted hemorrhagic transformation. Our study indicates that tumor hypoxia, following rapid tumor growth, may promote hemorrhagic transformation in pituitary adenomas via the HIF- $1 \alpha$ signaling pathway.
\end{abstract}

\section{Introduction}

Pituitary apoplexy is a rare but well described clinical syndrome resulting from hemorrhage or hemorrhagic infarction of a pre-existing pituitary adenoma (1). The presentation of this complication varies from asymptomatic to catastrophic. It is usually spontaneous, but it can also be induced by numerous

Correspondence to: Dr Ting Lei, Department of Neurosurgery, Tongji Hospital, Tongji Medical College, Huazhong University of Science and Technology, Jie Fang Avenue 1095, Wuhan 430030, P.R. China

E-mail: tlei@tjh.tjmu.edu

Key words: pituitary apoplexy, intratumoral hemorrhage, hypoxia inducible factor $1 \alpha$, vascular endothelial growth factor, BNIP3 precipitating factors $(2,3)$. The underlying mechanism of pituitary apoplexy is in infancy, and some theories have been proposed such as intratumor vasospasm, rapid tumor growth outstripping arterial supply, and fragile neovascularization (4).

Solid tumors are exposed to hypoxic conditions due to an imbalance between uncontrolled growth, impaired oxygen supply, and the accelerated consumption (5). The hypoxia inducible factor $1 \alpha$ (HIF-1 $\alpha)$ is a key regulator of hypoxia cellular adaptive responses. For this reason, nuclear expression of HIF-1 $\alpha$ reflects the hypoxic state of local tissue (6). Recently, HIF-1 $\alpha$ has been associated with various hemorrhagic events such as hemorrhagic infarction $(7,8)$, trauma hemorrhage (9), and hemorrhage in cerebral arteriovenous malformations (10). Mechanistic study revealed that HIF-1 $\alpha$ may promote hemorrhagic transformation via activating its downstream genes, including vascular endothelial growth factor (VEGF) and the proapoptotic gene BNIP3.

VEGF is a unique growth factor in the process of vasculogenesis and plays a vital role in modulating endothelial cell proliferation and vascular permeability, both of which contribute to instability of vessels, thereby predisposing to hemorrhagic events. Besides VEGF, the proapoptotic gene BNIP3 serves as a central regulator of cell death during hypoxia. Cell death, especially in endothelial cells, may lead to endothelial cell detachment, higher vascular permeability, and microvascular obstruction resulting in hemorrhagic incidents. Hence, previous studies suggest a possible role of HIF- $1 \alpha$-VEGF and HIF-1 $\alpha$-BNIP3 apoptosis pathways in the pathology of hemorrhagic transformation (11).

Since apoplectic pituitary adenomas display higher proliferation than non-apoplectic ones and HIF-1 $\alpha$ is expressed in all types of pituitary adenomas $(12,13)$, it is anticipated that fast growing tumor cells are prone to outstrip their blood supply and suffer sublethal local hypoxia, which itself stimulates HIF-1 $\alpha$ activity and then promotes hemorrhagic transformation in pituitary adenomas. Thus, in this study, we investigated cell proliferation and HIF- $1 \alpha$ signaling in apoplectic and non-apoplectic pituitary adenomas and evaluated their relation with pituitary apoplexy. Furthermore, the association between HIF-1 $\alpha$ signaling and hemorrhagic transformation was also identified using in vitro and in vivo approaches. Our results indicate that tumor hypoxia following rapid tumor growth may promote hemorrhagic transformation in pituitary adenomas via the HIF-1 $\alpha$ signaling pathway. 


\section{Materials and methods}

Cell lines. Since prolactinomas constitute the most prevalent hormone-secreting pituitary adenomas (14), we chose to use the MMQ rat tumor cell line in this study as it secretes only prolactin (15). Cells were maintained in F12 culture medium supplemented with 5\% fetal bovine serum (FBS), 10\% horse serum, penicillin $(100 \mu \mathrm{g} / \mathrm{ml})$ and streptomycin $(100 \mu \mathrm{g} / \mathrm{ml})$ in a humidified incubator $\left(37^{\circ} \mathrm{C}, 5 \%\right.$ carbon dioxide). The MMQ cell line that overexpresses HIF-1 $\alpha$ was established by the GeneChem Bio-Tech Co., Ltd (Shanghai). Briefly, the MMQ cells were transfected with pcDNA3 vector or HIF-1 $\alpha /$ pcDNA3 (GenBank no. NM_024359) encoding plasmid via lentiviral transduction. Transfected cells were selected with G418 and then cells overexpressing HIF-1 $\alpha$ (MMQ/HIF-1 $\alpha$ ) and vector control cells $(\mathrm{MMQ} / \mathrm{C})$ were generated. As shown in Fig. 3A and B, using this lentiviral vector-expressing GFP, $>90 \%$ of MMQ cells were stably transduced $(\mathrm{MOI}=20)$. For siRNA knockdown of HIF- $1 \alpha$, predesigned siRNAs targeting rat HIF-1 $\alpha$ and control siRNA duplex were purchased from Invitrogen. Specific or control siRNAs were transfected into the cells using Lipofectamine 2000 (Invitrogen) according to the manufacturer's protocol. The degree of knockdown was evaluated by real-time RT-PCR and Western blot analysis.

Patients and surgical specimens. Tissue samples from patients with apoplectic and non-apoplectic pituitary adenomas were used in the study. Diagnostic criteria for apoplectic pituitary adenomas were the occurrence of pituitary hemorrhage which was confirmed by preoperative magnetic resonance imaging (MRI), gross observation at surgery, and the postoperative histopathological findings. All patients received surgery between May 2009 to December 2010 at the Department of Neurosurgery, Tongji Hospital, Huazhong University of Science and Technology, Wuhan, China. There were 30 patients with apoplectic pituitary adenomas ( 6 men, 24 women; age range $17-62$ years, mean age $40.50 \pm 13.00$ years) and 30 patients with non-apoplectic ones ( 8 men, 22 women; age range $22-55$ years, mean age $37.50 \pm 9.94$ years). For each sample, the tissue was bisected, one half was frozen for protein and RNA extraction, the other half was fixed and embedded in paraffin for histology and immunohistochemisty studies. Informed consent was obtained from all patients in this study and the study protocol was approved by the local Ethics Committee.

Immunohistochemisty staining and hematoxylin and eosin $(H \& E)$ staining. Immunohistochemistry was performed using anti-HIF-1 $\alpha$ (Cell Signaling Technology); anti-PCNA (Santa Cruz Biotechnology); anti-VEGF (Santa Cruz Biotechnology); and anti-BNIP3 (Epitomics) antibodies. Briefly, for immunohistochemistry, the tissue sections were deparaffinized and hydrated by successive washes with xylene and ethanol. Endogenous peroxidase was blocked with $3 \%$ hydrogen peroxide. Slides were incubated with $10 \%$ normal serum followed by the primary antibodies incubated overnight at $4^{\circ} \mathrm{C}$. The slides were then incubated with biotinylated secondary antibody for $30 \mathrm{~min}$, followed by $\mathrm{ABC}$ reagent (Vector Labs) and diaminobenzidine. Sections were dehydrated and mounted. Digital images were obtained using an Olympus microscope equipped with a SPOT digital camera. For H\&E staining, the sections were deparaffinized and rehydrated in successive xylene and alcohol, and then stained with $\mathrm{H} \& \mathrm{E}$ for the morphological analysis.

Annexin V-PI staining and flow cytometric analysis. Cells were washed and suspended in $200 \mu \mathrm{l}$ binding buffer in the presence of $5 \mu \mathrm{l}$ Annexin V (mixed well) and $5 \mu 1$ PI for flow cytometry. For each sample, 10,000 cells were analyzed. Apoptotic (Annexin $\left.\mathrm{V}^{+}, \mathrm{PI}^{-}\right)$, necrotic $\left(\right.$Annexin $\left.\mathrm{V}^{+}, \mathrm{PI}^{+}\right)$and live (Annexin $\left.\mathrm{V}^{-}, \mathrm{PI}^{-}\right)$cells were expressed as percentages of the 10,000 cells. The apoptotic index was calculated by the upper right and lower right quadrants. Positive and negative controls were included and all samples were assayed in triplicate.

Tunel staining. Terminal deoxynucleotidyl transferase mediated dUTP-biotin nick end labeling (TUNEL) was performed according to the manufacturer's directions (Millipore). Briefly, the tissue sections were dewaxed, permeabilized and rinsed with PBS, pre-incubated in TdT reaction buffer, followed by incubation in TdT reaction mixture. Sections were washed with $\mathrm{dH}_{2} \mathrm{O}$ and PBS before treating with $0.3 \% \mathrm{H}_{2} \mathrm{O}_{2}$ in PBS for $30 \mathrm{~min}$. The sections were then treated with streptavidinHRP for $10 \mathrm{~min}$, DAB solution for $5 \mathrm{~min}$, dehydrated through a series of increasing alcohol solutions followed by xylene before mounting on the coverslips. Around 1,000 cells in five different fields consisting of TUNEL-positive cells were counted per coverslip under the microscope. Cell numbers were converted into percentage of apoptotic cells calculated from total cell numbers.

Western blot analysis. Nuclear or cytoplasmic protein was extracted and the concentration was determined using the Bradford assay (Bio-Rad). Proteins were electrophoresed and electroblotted onto nitrocellulose membranes. The membranes were blocked and incubated with primary antibodies [1:1,000 anti-HIF-1 $\alpha$ (Cell Signaling Technology), 1:1,000 anti-PCNA, 1:1,000 anti-VEGF, 1:3,000 anti-GAPDH (Santa Cruz Biotechnology) and 1:1,000 anti-BNIP3 (Epitomics)] overnight at $4^{\circ} \mathrm{C}$. Blots were then washed and incubated in horseradish peroxidase-conjugated secondary antibody $(1: 5,000)$ for $1.5 \mathrm{~h}$. The signal was detected using ECL substrate and quantified by densitometric analysis. Results were expressed as the ratio of intensity to that of control.

$R N A$ preparation and real-time $R T-P C R$. Total RNA was extracted using the RNeasy Mini kit (Qiagen). RNA (1 $\mu \mathrm{g})$ was subjected to reverse transcription using the Taqman reverse transcription kit. PCR reactions for all samples were performed in duplicate using $5 \mathrm{ng}$ complementary DNA, $12.5 \mu 12 \mathrm{X}$ SYBR Green Master Mix (Applied Biosystems), and $200 \mathrm{nM}$ each primer in a $25-\mu \mathrm{l}$ final volume. The PCR reactions were initiated with denaturation at $95^{\circ} \mathrm{C}$ for $10 \mathrm{~min}$; followed by 40 amplification cycles at $95^{\circ} \mathrm{C}$ for $15 \mathrm{sec}$ and $60^{\circ} \mathrm{C}$ for $1 \mathrm{~min}$. Samples were run in triplicate and results were averaged. The mRNA levels were normalized to GAPDH as outlined by the manufacturer. The primer sequences used for real-time RT-PCR were rat HIF-1 $\alpha$, F ATGACCACTGCTAAGGCAT CAGC, and R AGGTTAAGGCTCCTTGGATGAGC; rat VEGF, F TGTGCGGGCTGCTGCAATGAT and R TGTGCT 

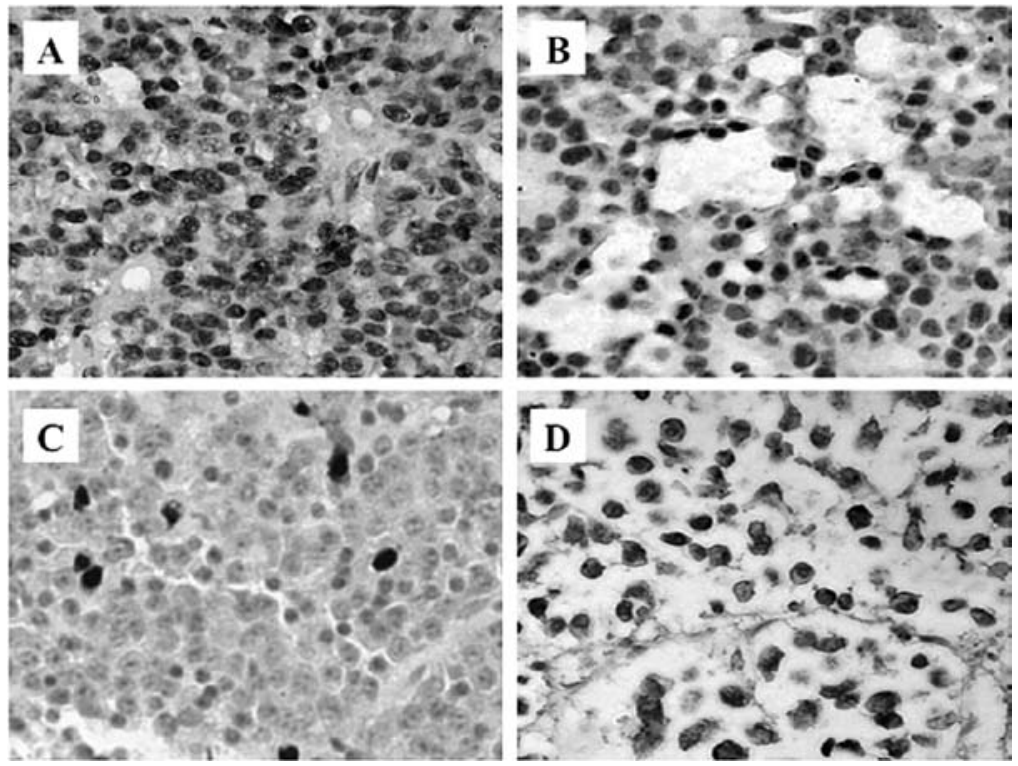

Figure 1. Immunoreactivity for (A) PCNA, (B) HIF-1 $\alpha$, (C) VEGF and (D) BNIP3 in human pituitary adenomas. (A) PCNA is strongly expressed in the cell nuclei of pituitary adenomas. (B) HIF-1 $\alpha$ is strongly expressed in the cell nuclei and cytoplasm of immunoreactive cells. (C) VEGF and (D) BNIP3, positive cytoplasmic immunoreactivity of adenomatous cells (original magnification, $\mathrm{x} 400$ ).

GGCTTTGGTGAGGTTTGA; rat GAPDH, F GTCTTCAC TACCATGGAGAAGG and R TCATGGATGACCTTGGC CAG; rat BNIP3, F ATTGGTCAAGTCGGCCAGAA and R AGTCGCTGTACGCTTTGGGT. The relative expression levels of the genes were calculated from the cycle threshold (Ct) value using the $\Delta \Delta \mathrm{Ct}$ method for quantification.

Nude mouse xenograft model. The animal experiments were conducted in accordance with NIH guidelines and with the approval of the local animal use committee. The mice were divided into three groups, five mice per group were included for the study. Six-week old athymic nude mice received subcutaneous implantations of $1 \times 10^{6} \mathrm{MMQ} / \mathrm{HIF}-1 \alpha$ cells, MMQ/C cells and MMQ cells in the lower rear flank, respectively. After 4 weeks, all mice were sacrificed and tumors were ressected for further analysis.

Statistical analysis. Statistical analysis was conducted using the SPSS 16.0 software. The Student's t-test was used to see whether genes and proteins were differentially expressed between adenomas with and without apoplexy. The Pearson correlation test was used to evaluate correlations. Statistical analysis in vitro and in vivo experiment was performed using the Student's t-test. P-values $<0.05$ were considered statistically significant.

\section{Results}

Apoptosis and expression of PCNA, HIF-1 $\alpha, V E G F$ and $B N I P 3$ in pituitary adenomas with and without hemorrhage. HIF- $1 \alpha$-VEGF and HIF- $1 \alpha$-BNIP3 apoptosis pathways have been associated with various hemorrhagic events. However, the involvement of these pathways in hemorrhagic transformation of pituitary adenomas is not understood. To verify this issue, we first assessed the activity of HIF-1 $\alpha$ signaling in pituitary adenomas with and without hemorrhage. The expres- sion of PCNA, HIF-1 $\alpha$, VEGF and BNIP3 was evaluated in 60 pituitary adenomas using immunohistochemistry and Western blot analysis. Furthermore, the percentages of apoptotic cells were analyzed by TUNEL staining.

In our study HIF-1 $\alpha$ staining, for the most part, was restricted to nuclei, however, traces of HIF-1 $\alpha$ expression was also observed in the cytoplasm (Fig. 1). PCNA was expressed in the nuclei of tumor cells. Staining for VEGF and BNIP3 was restricted to the cytoplasm. The extent, intensity, and distribution pattern of these proteins were heterogeneous even in the same section. Interestingly, some of the endothelial cells of tumor blood vessels also showed positive staining for these proteins and apoptotic features (Fig. 2C). Western blot results showed that apoplectic pituitary adenomas had significantly higher expression levels of PCNA, HIF-1 $\alpha$, VEGF and BNIP3 compared with non-apoplectic adenomas $(\mathrm{P}<0.05)$ (Fig. 2A and $\mathrm{B})$. The same trend was also seen in TUNEL staining (hemorrhagic tumors vs. non-hemorrhagic ones, $4.20 \pm 1.10 \%$ vs. $0.30 \pm 0.12 \%, \mathrm{P}<0.05$ ) (Fig. $2 \mathrm{D}$ ). The expression of HIF- $1 \alpha$ was associated with that of PCNA $(\mathrm{R}=0.674, \mathrm{P}=0.001)$. In addition, based on the quantitative Western blot data, the Spearman correlation coefficients for VEGF and BNIP3 protein vs. HIF-1 $\alpha$ protein expression in pituitray adenomas were 0.571 $(\mathrm{P}<0.05)$ and $0.480(\mathrm{P}<0.05)$, respectively, indicating that expression of HIF-1 $\alpha$ correlated positively with VEGF and BNIP3 in pituitary adenomas. These data demonstrate that tissue hypoxia as shown by localized HIF-1 $\alpha$ expression in fast growing adenomas may be associated with pituitary adenoma hemorrhage.

Overexpression of HIF-1 $\alpha$ activates its target genes VEGF and $B N I P 3$ in $M M Q$ cells. In order to define HIF-1 $\alpha$-VEGF and HIF-1 $\alpha$-BNIP3 apoptosis pathways in pituitary tumor cells, we further established a stable MMQ cell line overexpressing HIF-1 $\alpha$ (MMQ/HIF-1 $\alpha)$ and a stable MMQ cell line transfected with pCDNA3 vector $(\mathrm{MMQ} / \mathrm{C})$ to serve as a control. Western 
A

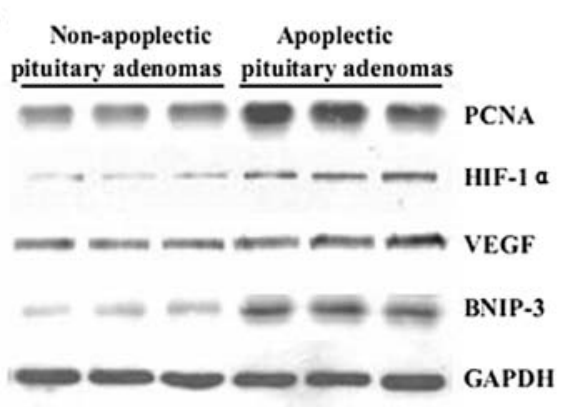

C

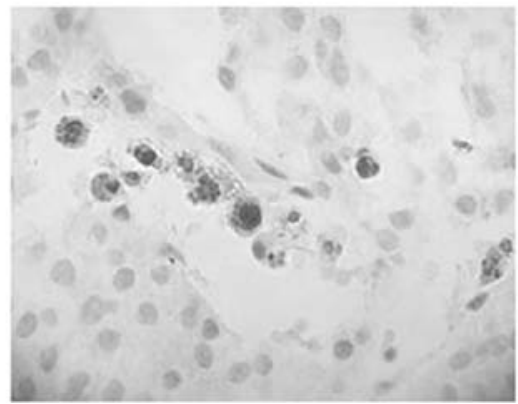

B

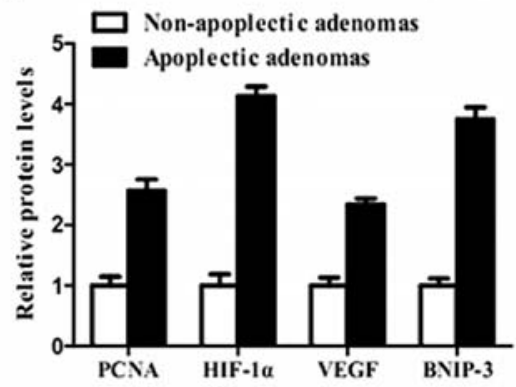

D

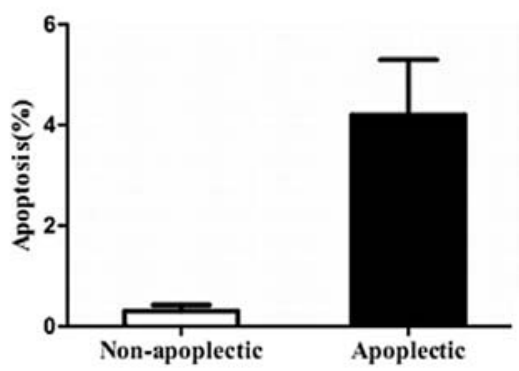

Figure 2. Apoptosis, PCNA, HIF-1 $\alpha$, VEGF and BNIP3 expression in apoplectic and non-apoplectic pituitary adenomas. (A and B) Western blot analysis shows increased expression of PCNA, HIF-1 $\alpha$, VEGF and BNIP3 protein expression in apoplectic pituitary adenomas, compared with non-apoplectic ones. GAPDH served as internal control. (C) Representative imaging of TUNEL staining of endothelial cells in pituitary adenomas (original magnification, $\mathrm{x} 400$ ). (D) Bar graph shows that apoplectic pituitary adenomas display increased TUNEL positive cells, compared with non-apoplectic ones. The percentage of apoptosis represents the mean $\pm \mathrm{SD}$ of all samples.

A

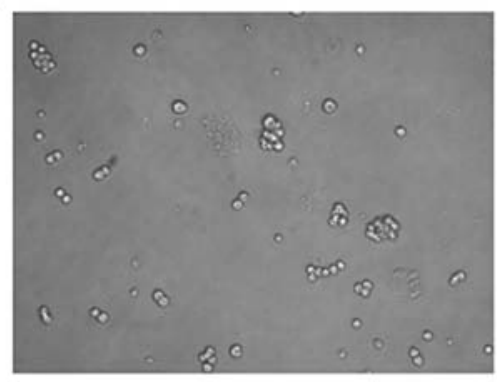

Light Microscope

C

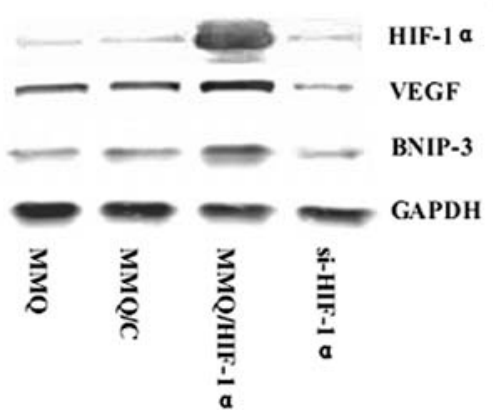

B

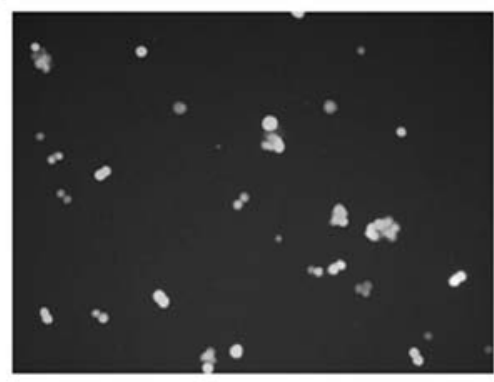

Fluo Microscope

D

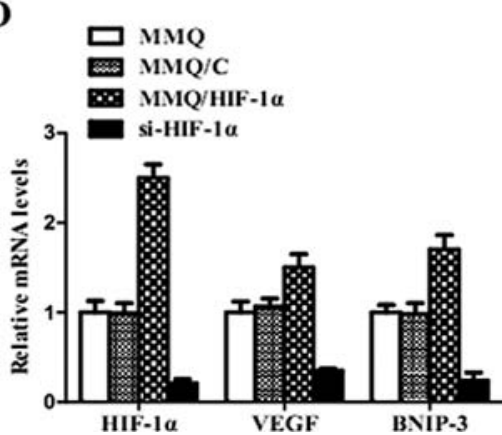

Figure 3. Effect of HIF-1 $\alpha$ transfection or knockdown on VEGF and BNIP3 expression in MMQ cells. (A and B) Fluoresence microscopy showed that $>90 \%$ of MMQ cells were transduced by lentiviral vector-expressing GFP. (C) Western blot analysis and (D) real-time RT-PCR showed that lentivirus-mediated overexpression of HIF-1 $\alpha$ (MMQ/HIF-1 $\alpha$ ) increased expression levels of VEGF and BNIP3 in MMQ cells, whereas siRNA-mediated knockdown of HIF-1 $\alpha$ (si-HIF-1 $\alpha$ ) had the opposite effect. GAPDH served as an internal control.

blot analysis and real-time RT-PCR was performed to address whether increased level of HIF-1 $\alpha$ could modulate its target genes VEGF and BNIP3 in pituitary adenoma cells. As shown in Fig. 3C and D, MMQ/HIF-1 $\alpha$ cells exhibited much higher protein and mRNA levels of VEGF and BNIP3 than those in $\mathrm{MMQ} / \mathrm{C}$ cells. Furthermore, a higher apoptosis rate was found 
A
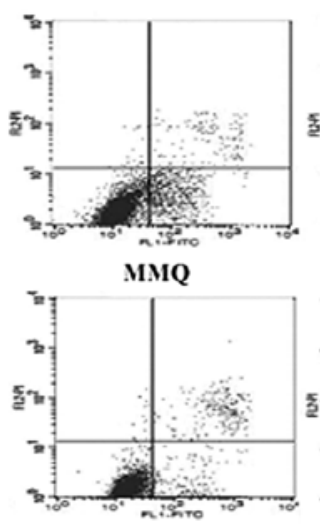

MMQ/HIF-1 a

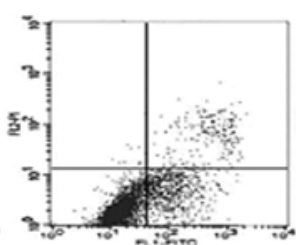

$\mathrm{MMQ} / \mathrm{C}$

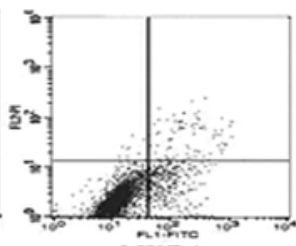

si-HIF-1 a
B

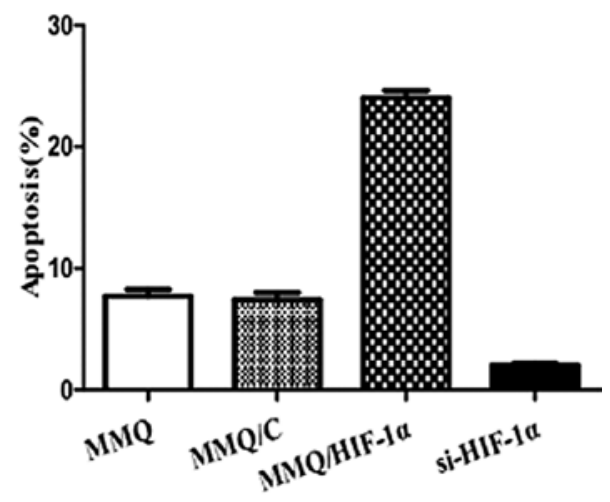

Figure 4. Effect of HIF-1 $\alpha$ transfection or knockdown on apoptosis in MMQ cells. (A) Annexin V-PI staining and flow cytometric analysis of apoptotic cells in MMQ/HIF-1 $\alpha$ cells, MMQ/C cells, MMQ cells and si-HIF-1 $\alpha$ MMQ cells. Lower right quadrant and upper right quadrant represents apoptotic cells. (B) Bar graph shows that, compared with other groups, MMQ/HIF-1 $\alpha$ cells display increased apoptotic cell rate, whereas si-HIF-1 $\alpha$ MMQ cells display decreased apoptotic cell rate.

A

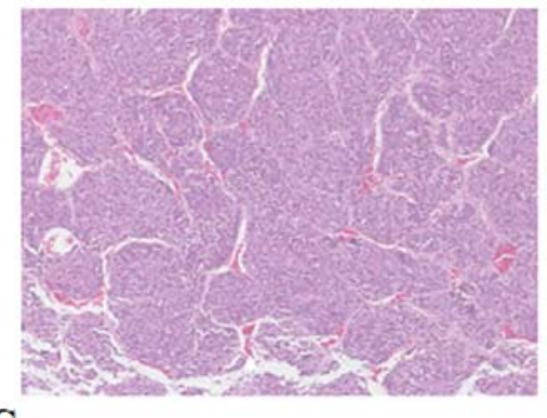

C

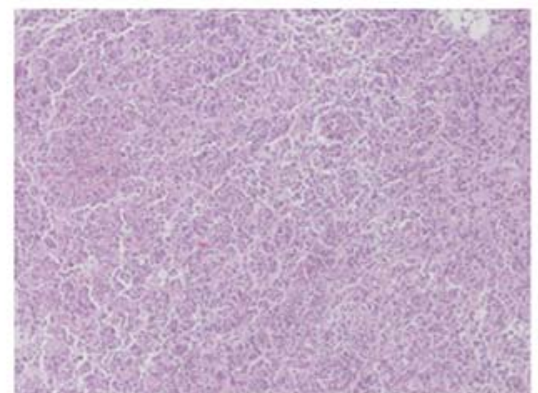

B

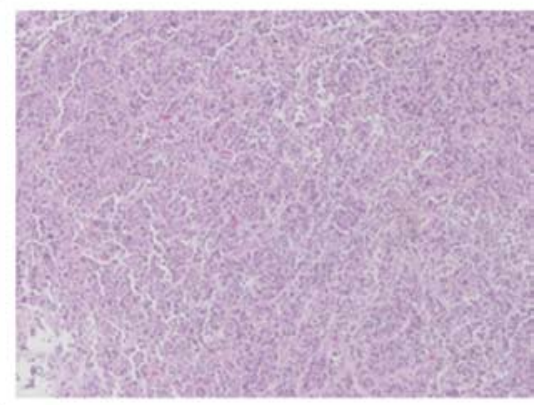

D

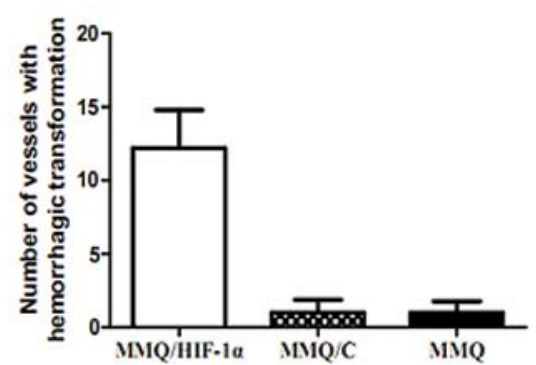

Figure 5. Effect of HIF-1 $\alpha$ transfection on hemorrhagic transformation of MMQ cell xenografts. (A, B and C) Representative H\&E staining pictures of MMQ/ HIF-1 $\alpha, M M Q / C$ and MMQ cell xenografts, respectively. (D) Bar graph shows that xenografts of MMQ/HIF-1 $\alpha$ group display increased vascular leak, areas of extensive hemorrhage and accumulation of serum proteins, compared with those of other groups.

in MMQ/HIF-1 $\alpha$ cells, as shown by flow cytometry (Fig. 4). Our result suggests that overexpression of HIF-1 $\alpha$ activates its target genes VEGF and BNIP3 in MMQ cells.

Knockdown of HIF-1 $\alpha$ reduces the VEGF and BNIP3 expression in $M M Q$ cells. As we have shown HIF-1 $\alpha$ stimulates VEGF and BNIP3 expression, we hypothesized that blocking HIF-1 $\alpha$ activity would inhibit VEGF and BNIP3 expression in MMQ cells and using siRNA we knocked down HIF-1 $\alpha$ in the MMQ cells and measured apoptosis levels, VEGF and BNIP3 expression. We found that siRNA-mediated knockdown of HIF-1 $\alpha$ led to reduced apoptosis, VEGF and BNIP3 expression levels in MMQ cells (Figs. 3C and D and 4), whereas control
siRNA had no effect on MMQ cells (data not shown). Taken together, these data establish the presence of HIF-1 $\alpha$-VEGF and HIF-1 $\alpha$-BNIP3 apoptosis pathways in MMQ pituitary adenoma cells.

HIF-1 $\alpha$ overexpression causes hemorrhagic transformation in $M M Q$ cell xenografts. To determine whether overexpression of HIF-1 $\alpha$ causes hemorrhagic transformation in pituitary adenomas, we implanted the nude mice subcutaneously with MMQ/HIF-1 $\alpha$, MMQ/C and MMQ cells, respectively. After 4 weeks, tumor size did not differ significantly in MMQ/HIF-1 $\alpha$ xenografts compared with MMQ/C and MMQ xenografts. Compared with the control and MMQ/C cell groups, xeno- 
A

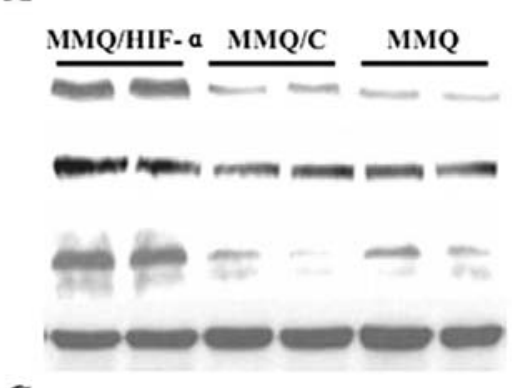

C

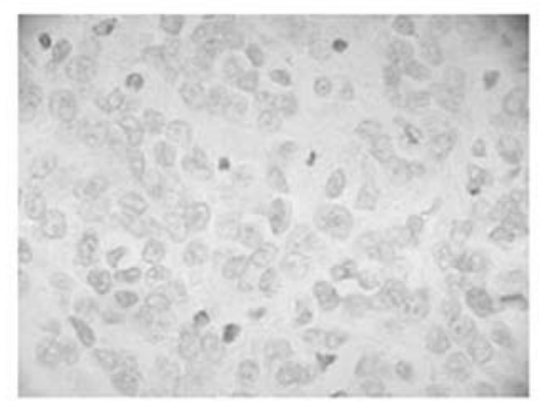

B

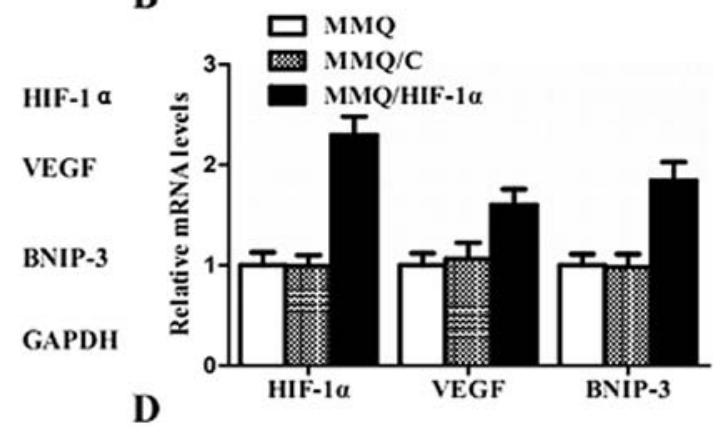

D

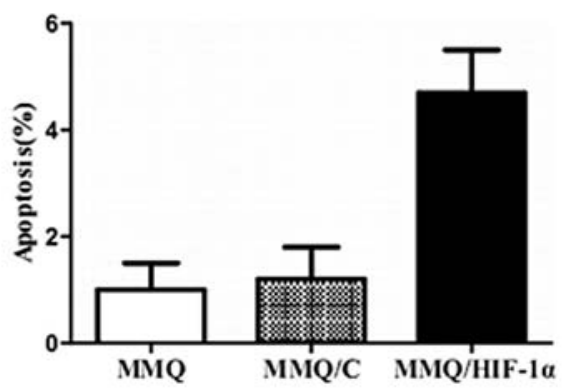

Figure 6. Apoptosis, HIF-1 $\alpha$, VEGF and BNIP3 expression in MMQ cell xenografts. Western blot (A) and real-time RT-PCR (B) analysis of tissue extracts derived from xenografts of MMQ/HIF-1 $\alpha, \mathrm{MMQ} / \mathrm{C}$ and control MMQ cells. GAPDH served as an internal control. (C) Representative imaging of TUNEL staining in MMQ/ HIF-1 $\alpha$ xenografts (original magnification, $\mathrm{x} 400$ ). (D) Bar graph shows that MMQ/ HIF-1 $\alpha$ xenografts displayed higher TUNEL positive cells rate, compared with the other groups. The percentage of apoptosis is shown as the mean \pm SD of all samples.

grafts of MMQ/HIF-1 $\alpha$ groups displayed increased vascular leak, areas of extensive hemorrhage and accumulation of serum proteins, as shown by H\&E staining (Fig. 5). Vessel numbers with hemorrhagic transformation were significantly greater in the MMQ/HIF-1 $\alpha$ group than in the other groups (12.2 \pm 2.6 vs. $1.0 \pm 0.9$ and $1.0 \pm 0.8 ; \mathrm{P}<0.05)$, suggesting significant greater hemorrhagic transformation rate in MMQ/HIF-1 $\alpha$ cell xenografts. These observations suggest that overexpression of HIF-1 $\alpha$ induces hemorrhagic transformation in MMQ cell xenografts.

Accumulation of HIF-1 $\alpha$ activates its target genes VEGF and BNIP3 in MMQ cell xenografts. We further verified the involvement of the HIF- $1 \alpha / \mathrm{VEGF} / \mathrm{BNIP} 3$ signaling axis in hemorrhagic transformation of the MMQ cell xenografts in nude mice. As shown by Western blot analysis, real-time RT-PCR and TUNEL staining (Fig. 6), xenografts of the MMQ/HIF-1 $\alpha$ group displayed elevated levels of HIF-1 $\alpha$, VEGF, BNIP3 and apoptosis (MMQ/HIF-1 $\alpha$ xenografts vs. MMQ/C and MMQ ones, $4.70 \pm 1.40$ vs. $1.2 \pm 0.24$ and $1.0 \pm 0.21 \%, \mathrm{P}<0.05$ ). These in vivo results are consistent with our in vitro data. Taken together, our data provide the evidence that the HIF- $1 \alpha$-VEGF and HIF-1 $\alpha$-BNIP3 apoptosis pathways may be involved in hemorrhagic transformation of MMQ cell xenografts.

\section{Discussion}

To the best of our knowledge, this is the first report documenting the functional involvement of the HIF-1 $\alpha$ signaling pathway in hemorrhagic transformation of pituitary adenomas. Our study reveals a key role of HIF-1 $\alpha$ signaling in hemorrhagic transformation of pituitary adenomas using a pituitary adenoma cell xenograft model overexpressing HIF-1 $\alpha$. We showed that tissue hypoxia, co-existent with rapid tumor growth, promotes hemorrhagic transformation in pituitary adenomas via HIF-1 $\alpha$ VEGF and HIF-1 $\alpha$-BNIP3 apoptosis pathways.

Local rapid cellular growth is a very common event in solid tumors. It often results in local hypoxia due to the inability of local vasculature to supply enough oxygen and nutrients to rapidly dividing tumor cells (5). The resultant hypoxia may inhibit cell division or even lead to cell death (16). Emerging evidence suggests that HIF-1 $\alpha$ plays a pivotal role in the hypoxic responses and its presence often indicates hypoxia in tissues (6). In addition, HIF-1 $\alpha$ has been reported to be present in all types of pituitary adenomas, indicating that HIF-1 $\alpha$ may play a role in the development of pituitary adenomas (13). Since fast growing tumor cells often aggravate hypoxia in local tissues and PCNA is a reliable marker for cell proliferation (16-18), we analyzed PCNA and HIF-1 $\alpha$ expression in hemorrhagic and non-hemorrhagic human pituitary adenoma tissues. It was observed that expression of HIF-1 $\alpha$ and PCNA was closely related to intratumoral hemorrhage in pituitary adenoma tissues. Moreover, there was a positive correlation between the expression of HIF- $1 \alpha$ and PCNA. Based on these observations, we speculated that local tissue hypoxia may play a role in the development of hemorrhagic transformation in pituitary adenomas. To validate our hypothesis, we used MMQ cell xenotransplantation as an in vivo pituitary prolactinoma model in our study. Compared with controls, implantation of cells overexpressing HIF-1 $\alpha$ displayed obvious hemorrhagic transformation. Based on these findings, HIF-1 $\alpha$ seems to be an important factor in the development of intratumoral hemorrhage in pituitary adenomas. We deduced that local fast growing tumor cells tend to outstrip their blood supply and predispose themselves to sublethal hypoxia, which in turn activate HIF-1 $\alpha$ activity leading to hemorrhagic transformation in pituitary adenomas. 
Although our data showed a positive correlation between HIF-1 $\alpha$ activity and intratumoral hemorrhage in pituitary adenomas, the precise underlying mechanisms remained unclear. To date, HIF- $1 \alpha$ regulates a wide array of genes in response to hypoxia including VEGF and BNIP3 $(19,20)$, which are associated with various hemorrhagic events. VEGF not only increases vascular permeability, but also induces neovascularization, thereby increasing the risk for hemorrhagic transformation $(21,22)$. VEGF overexpression in U87 MG cells and U251 MG cells caused hemorrhagic tumors in nude mice $(23,24)$. Exogenous VEGF aggravated hemorrhagic transformation in rats after transient focal cerebral ischemia. In terms of pituitary adenomas, Arita et al (25) found a positive relation between hemorrhage and the protein expression of VEGF in 39 pituitary adenomas. However, no significant relationship between hemorrhage and VEGF immunoreactivity in pituitary adenomas was found in other studies (26-28). We speculate that the discrepancy may be due to the differences in sample size and the types of hormone-secreting tumors examined.

In addition to VEGF, the pro-apoptotic gene BNIP3 is dramatically induced by hypoxia and plays a central role in hypoxia-induced apoptosis and necrosis (29). Cell death, especially in endothelial cells, may lead to endothelial cell detachment, increased vascular permeability and microvascular obstruction, thereby triggering and/or aggravating hemorrhagic transformation (30). This is partially exemplified by increased apoptosis in the human ruptured aneurysm wall (31).

Based on the premise, in order to understand the mechanisms involved in hemorrhagic transformation of pituitary adenomas, we investigated apoptosis levels, VEGF and BNIP3 expression in our study. As expected, MMQ cells overexpressing HIF-1 $\alpha$ displayed elevated apoptosis, enhanced expression of VEGF and BNIP3, whereas siRNA-mediated knockdown of HIF-1 $\alpha$ had the opposite effect. Besides the above, compared with non-hemorrhagic ones, human hemorrhagic adenomas also displayed moderate correlation between HIF-1 $\alpha$ and VEGF or BNIP3. In the present study, we documented the possible involvement of HIF-1 $\alpha$-VEGF and HIF-1 $\alpha$-BNIP3 apoptosis pathways in hemorrhagic transformation of pituitary adenomas. There is research going on in our lab to fully understand the involvement of HIF-1 $\alpha /$ VEGF/ BNIP3 signal axis in the development of intratumoral hemorrhage in pituitary adenomas.

In conclusion, this study demonstrates that pituitary adenomas with accelerated growth outstripping blood supply tended to develop intratumoral hemorrhage in pituitary adenomas and that HIF-1 $\alpha$-VEGF and HIF-1 $\alpha$-BNIP3 apoptosis pathways may be involved in mediating the occurrence of hemorrhagic transformation.

\section{Acknowledgements}

This study was supported by a grant from the National Natural Science Foundation of China (no. 30672161).

\section{References}

1. Semple PL, Webb MK, de Villiers JC and Laws ER Jr: Pituitary apoplexy. Neurosurgery 56: 65-73, 2005.
2. Biousse V, Newman NJ and Oyesiku NM: Precipitating factors in pituitary apoplexy. J Neurol Neurosurg Psychiatry 71: 542-545, 2001.

3. Semple PL, Jane JA Jr and Laws ER Jr: Clinical relevance of precipitating factors in pituitary apoplexy. Neurosurgery 61: 956-962, 2007

4. Chang CV, Felicio AC, Toscanini AC, Teixeira MJ and CunhaNeto MB: Pituitary tumor apoplexy. Arq Neuropsiquiatr 67: 328-333, 2009.

5. Vaupel P, Kallinowski F and Okunieff P: Blood flow, oxygen consumption and tissue oxygenation of human tumors. Adv Exp Med Biol 277: 895-905, 1990.

6. Ryan HE, Poloni M, McNulty W, et al: Hypoxia-inducible factor1alpha is a positive factor in solid tumor growth. Cancer Res 60: 4010-4015, 2000.

7. Chen C, Ostrowski RP, Zhou C, Tang J and Zhang JH: Suppression of hypoxia-inducible factor-1alpha and its downstream genes reduces acute hyperglycemia-enhanced hemorrhagic transformation in a rat model of cerebral ischemia. J Neurosci Res 88: 2046-2055, 2010.

8. Zhou D, Matchett GA, Jadhav V, Dach N and Zhang JH: The effect of 2-methoxyestradiol, a HIF-1 alpha inhibitor, in global cerebral ischemia in rats. Neurol Res 30: 268-271, 2008.

9. Hsieh CH, Nickel EA, Hsu JT, Schwacha MG, Bland KI and Chaudry IH: Trauma-hemorrhage and hypoxia differentially influence kupffer cell phagocytic capacity: role of hypoxiainducible-factor-1alpha and phosphoinositide 3-kinase/Akt activation. Ann Surg 250: 995-1001, 2009.

10. Ng I, Tan WL, Ng PY and Lim J: Hypoxia inducible factor1alpha and expression of vascular endothelial growth factor and its receptors in cerebral arteriovenous malformations. J Clin Neurosci 12: 794-799, 2005.

11. Yan J, Chen C, Lei J, et al: 2-methoxyestradiol reduces cerebral vasospasm after 48 hours of experimental subarachnoid hemorrhage in rats. Exp Neurol 202: 348-356, 2006.

12. Liu ZH, Chang CN, Pai PC, et al: Clinical features and surgical outcome of clinical and subclinical pituitary apoplexy. J Clin Neurosci 17: 694-699, 2010.

13. Vidal S, Horvath E, Kovacs K, Kuroki T, Lloyd RV and Scheithauer BW: Expression of hypoxia-inducible factor-1alpha (HIF-1alpha) in pituitary tumours. Histol Histopathol 18: 679-686, 2003.

14. Aron DC, Tyrrell JB and Wilson CB: Pituitary tumors. Current concepts in diagnosis and management. West J Med 162: 340-352, 1995.

15. Judd AM, Login IS, Kovacs K, et al: Characterization of the MMQ cell, a prolactin-secreting clonal cell line that is responsive to dopamine. Endocrinology 123: 2341-2350, 1988.

16. Schmaltz C, Hardenbergh PH, Wells A and Fisher DE: Regulation of proliferation-survival decisions during tumor cell hypoxia. Mol Cell Biol 18: 2845-2854, 1998.

17. Daidoji H, Takasaki Y and Nakane PK: Proliferating cell nuclear antigen (PCNA/cyclin) in plant proliferating cells: immunohistochemical and quantitative analysis using autoantibody and murine monoclonal antibodies to PCNA. Cell Biochem Funct 10: 123-132, 1992.

18. Turner HE and Wass JA: Are markers of proliferation valuable in the histological assessment of pituitary tumours. Pituitary 1: 147-151, 1999.

19. Cummins EP and Taylor CT: Hypoxia-responsive transcription factors. Pflugers Arch 450: 363-371, 2005.

20. Carmeliet P, Dor Y, Herbert JM, et al: Role of HIF-1alpha in hypoxia-mediated apoptosis, cell proliferation and tumour angiogenesis. Nature 394: 485-490, 1998.

21. Dvorak HF, Nagy JA, Feng D, Brown LF and Dvorak AM: Vascular permeability factor/vascular endothelial growth factor and the significance of microvascular hyperpermeability in angiogenesis. Curr Top Microbiol Immunol 237: 97-132, 1999.

22. Nagy JA, Benjamin L, Zeng H, Dvorak AM and Dvorak HF: Vascular permeability, vascular hyperpermeability and angiogenesis. Angiogenesis 11: 109-119, 2008.

23. Cheng SY, Nagane M, Huang HS and Cavenee WK: Intracerebral tumor-associated hemorrhage caused by overexpression of the vascular endothelial growth factor isoforms VEGF121 and VEGF165 but not VEGF189. Proc Natl Acad Sci USA 94: 12081-12087, 1997.

24. Ke LD, Shi YX and Yung WK: VEGF(121), VEGF(165) overexpression enhances tumorigenicity in U251 MG but not in NG-1 glioma cells. Cancer Res 62: 1854-1861, 2002. 
25. Arita K, Kurisu K, Tominaga A, et al: Relationship between intratumoral hemorrhage and overexpression of vascular endothelial growth factor (VEGF) in pituitary adenoma. Hiroshima J Med Sci 53: 23-27, 2004.

26. Fukui S, Otani N, Nawashiro H, et al: The association of the expression of vascular endothelial growth factor with the cystic component and haemorrhage in pituitary adenoma. J Clin Neurosci 10: 320-324, 2003.

27. Takada K, Yamada S and Teramoto A: Correlation between tumor vascularity and clinical findings in patients with pituitary adenomas. Endocr Pathol 15: 131-139, 2004.
28. Fukui S, Nawashiro H, Otani N, et al: Vascular endothelial growth factor expression in pituitary adenomas. Acta Neurochir Suppl 86: 519-521, 2003.

29. Guo K, Searfoss G, Krolikowski D, et al: Hypoxia induces the expression of the pro-apoptotic gene BNIP3. Cell Death Differ 8: 367-376, 2001.

30. Dimmeler S and Zeiher AM: Endothelial cell apoptosis in angiogenesis and vessel regression. Circ Res 87: 434-439, 2000.

31. Pentimalli L, Modesti A, Vignati A, et al: Role of apoptosis in intracranial aneurysm rupture. J Neurosurg 101: 1018-1025, 2004. 